Article

\title{
Antioxidant Potential of Polyphenols and Tannins from Burs of Castanea mollissima Blume
}

\author{
Shan Zhao, Jie Yuan Liu, Si Yu Chen, Ling Ling Shi, Yu Jun Liu * and Chao Ma * \\ National Engineering Laboratory for Tree Breeding, College of Biological Sciences and \\ Biotechnology, Beijing Forestry University, Beijing 100083, China \\ * Authors to whom correspondence should be addressed; E-Mails: machao@bjfu.edu.cn (C.M.); \\ yjliubio@163.com (Y.J.L.); Tel.: +86-10-62337855; Fax: +86-10-62337855.
}

Received: 31 August 2011; in revised form: 29 September 2011 / Accepted: 29 September 2011 / Published: 12 October 2011

\begin{abstract}
Spiny burs of Castanea mollissima Blume (Chinese chestnut) are usually discarded as industrial waste during post-harvesting processing. The objective of this study was to establish an extraction and isolation procedure for tannins from chestnut burs, and to assess their potential antioxidant activity. Aqueous ethanol solution was used as extraction solvent, and HPD 100 macroporous resin column was applied for isolation. The influence of solvent concentration in the extraction and elution process on extraction yield, tannins and polyphenols content, as well as antioxidant potential, including DPPH and ABTS radical scavenging ability, reducing power ability and cellular antioxidant ability were assessed. In both the extraction and isolation process, $50 \%$ aqueous ethanol led to superior total tannins and polyphenols content as well as significantly higher antioxidant activity. In addition, the antioxidant activity and the total tannins content in extracts and fractions had a positive linear correlation, and the predominant components responsible for antioxidant activities were characterized as hydrolysable tannins. To the best of our knowledge, this is the first report on the enrichment of tannins from burs of C. mollissim using macroporous resin chromatography, and to assess the cellular antioxidant activity of them.
\end{abstract}

Keywords: Castanea mollissima; polyphenols; tannins; antioxidant; chestnut burs 


\section{Introduction}

Chinese chestnut (Castanea mollissima Blume) belongs to the Castanea family and is a wood plant widely cultivated in Europe, North American and Asia as an economic crop. Its sweet kernels are edible and have been used in traditional Chinese medicine for treatment of gastroenteritis, bronchitis, and regurgitation [1]. In China, Chinese chestnuts are widely cultivated in Hebei, Shandong, Hunan, Jiangxi, Fujian and Anhui provinces, with a total annual kernel production of up to 460,000 t, contributing to approximately $60 \%$ of the global production. Chestnut burs with 1 to $2 \mathrm{~cm}$ long, $1 \mathrm{~mm}$ thick spines, representing about $20 \%$ of the total chestnut dry weight, are byproducts of chestnut cultivation, usually being discarded upon harvesting. Therefore, the use of chestnut burs as potential source of natural antioxidants and functional food ingredients is of great interest to the chestnut industries.

Over the past few years, much attention has been paid to natural polyphenols such as silymarin, curcumin, green tea, and grape seed extracts [2], which could provide protection against the harmful effects of free radicals and reduce the risk of several diseases, including cancer [3,4] and inflammation [5], as well as to modulate immune responsiveness [6]. Although some papers have been published regarding the antioxidant activity and phenolic constituents of chestnut seed shells [7,8] and kernels [9], little is known about the constituents and bioactivities of Chinese chestnuts burs. Recently, Vázquez and co-workers $[10,11]$ did lots of valuable studies on the polyphenols from burs of $C$. sativa. They found that the extract from C. sativa burs exhibited antioxidant potential in the DPPH (1,1-diphenyl-2picrylhydrazyl), $\operatorname{ABTS}^{+}$(2,2'-azinobis-3-ethylbenzothiazoline-6-sulfonic acid) radicals and reducing power assays. Mujić and co-workers also reported that the spiny burs extracts of $C$. sativa could increase rat pancreatic $\beta$-cell viability after streptozotocin treatment by protecting DNA from oxidative damage and by enhancing the natural antioxidant system [12]. To obtain the polyphenols from the burs of C. sativa., Vázquez tried several extraction solvents, and found that the extraction yield, the total polyphenols content and antioxidant activity of extracts decreased with the polarity of the solvent [10], and recommended ethanol as the extraction solution for food or pharmaceutical application due to its GRAS (General Recognized as Safe) status [11]. However, the polyphenols content of crude extract was only $26.32 \mathrm{~g}$ gallic acid equivalent (GAE)/100 g extract, which was not good enough for further studies and industrial applications. Therefore, there was still an urgent need to establish an efficient and low cost method to enrich the polyphenols and tannins in extract. Thus, the objective of this study was to establish such a procedure for extraction and isolation, as well as to assess the antioxidant activity of the resulting extracts.

\section{Results and Discussion}

\subsection{Extraction of Polyphenols and Tannins}

The polyphenols and tannins contents of different extracts are presented in Table 1. The data therein shows that the yield of extract decreased significantly with increasing ethanol concentration. The extraction with water led to the highest extraction yield, followed by $30 \%$ ethanol, $50 \%$ ethanol and $75 \%$ ethanol. This trend was consistent with the report of Vázquez [10], who measured the extracting yield from $C$. saiva burs using four different extraction solvents including acetone, ethanol, methanol, and water, and found that the extraction yield decreased as the polarity of the solvent decreased. 
The total polyphenols and tannins content in crude extract obtained using different solvents showed significant difference. As presented in Table 1, 50\% ethanol extracts showed the highest total polyphenols and tannins content, followed by $75 \%, 30 \%$ ethanol and water extracts. This indicated that water extracts contained much more non-phenols or non-tannins, which would make it much more difficult for the following purification of polyphenols and tannin. With respect to total polyphenols yields from raw burs, $75 \%$ ethanol extraction was the lowest, and there was no significant difference between water, 30\% ethanol and 50\% ethanol extract. Meanwhile, the 50\% ethanol extraction led to both highest total tannins content in extracts and highest yield of tannins from raw burs. Therefore, $50 \%$ ethanol was selected as the optimal solvent for extraction.

Table 1. Extracting yield and total polyphenols and tannins contents in chestnut burs extract.

\begin{tabular}{lllll}
\hline Gallic Acid Equivalent (GAE) & Water & $\mathbf{3 0 \%}$ Ethanol & $\mathbf{5 0 \%}$ Ethanol & $\mathbf{7 5 \%}$ Ethanol \\
\hline yield g / 100 g bur & $16.28 \pm 0.69^{\mathrm{A}}$ & $13.60 \pm 0.93^{\mathrm{B}}$ & $11.67 \pm 0.76^{\mathrm{C}}$ & $6.62 \pm 0.53^{\mathrm{D}}$ \\
Total polyphenols g GAE / 100 g Extract & $43.75 \pm 1.62^{\mathrm{A}}$ & $48.20 \pm 1.25^{\mathrm{B}}$ & $60.00 \pm 1.49^{\mathrm{C}}$ & $50.00 \pm 1.15^{\mathrm{B}}$ \\
Total polyphenols g GAE / 100 g bur & $7.12 \pm 0.89^{\mathrm{A}}$ & $6.56 \pm 0.76^{\mathrm{A}}$ & $7.00 \pm 1.02^{\mathrm{A}}$ & $3.31 \pm 0.62^{\mathrm{B}}$ \\
Total tannins g GAE / 100 g extract & $27.00 \pm 1.9^{\mathrm{A}}$ & $28.40 \pm 1.09^{\mathrm{A}}$ & $44.00 \pm 1.34^{\mathrm{B}}$ & $36.60 \pm 1.26^{\mathrm{C}}$ \\
Total tannins g GAE / 100 g bur & $4.39 \pm 0.54^{\mathrm{A}}$ & $3.86 \pm 0.39^{\mathrm{B}}$ & $5.13 \pm 0.43^{\mathrm{C}}$ & $2.42 \pm 0.51^{\mathrm{D}}$ \\
\hline \multicolumn{4}{c}{ A,B,C,D In each line with different letters means significant differences $(p<0.05)}$.
\end{tabular}

\subsection{Isolation of Total Polyphenols and Tannins}

To avoid the toxic organic solvents used in conventional separation techniques such as gel and high-speed counter-current chromatographic separation process and to increase the safety of the process, the adsorption and desorption on macroporous resins were utilized for isolation of chestnut bur polyphenols and tannins. In fact, macroporous resins have been successfully applied to the separation and isolation of effective components from many natural products, as an efficient method with a moderate purification effect, high absorption capacity, low operating costs, low solvent consumption, and easy regeneration [13-15]. After macroporous resin chromatography, the 50\% ethanol extracts of chestnut burs were fractionated into two parts, Fraction I washed out with water and Fraction II eluted with 50\% ethanol. As presented in Table 2, the polyphenols and tannins contents in Fraction II were approximately 2.5- and 4.6-fold higher than those in Fraction I, and 1.34- and 1.39-fold higher than those in crude extract.

Plant tannins constitute a complex group of naturally occurring polymers, and a rigorous chemical definition is difficult. Thus, tannins can conveniently be divided into two groups based on their structures, hydrolysable and condensed tannins [16]. To identify the predominant tannins in chestnut burs crude extracts and fractions, hydrolysable or condensed tannins, the content of condensed tannins was determined. As illustrated in Table 2, the content of condensed tannins was very low both in extracts and fractions, contributing less than $2 \%$ to the total tannins, which indicated that the predominant tannins in chestnut burs were hydrolysable tannins. This result was highly consistent with the report of Vázquez [10], who characterized the polyphenols of $C$. sativa burs as hydrolysable gallotannins based on MALDI-TOF spectrum. 
Table 2. Total polyphenols and tannins content in fractions.

\begin{tabular}{llll}
\hline & Crude extract & Fraction II & Fraction I \\
\hline Total polyphenols (mg GAE / g) & $600.0 \pm 14.9^{\mathrm{A}}$ & $801.4 \pm 15.6^{\mathrm{B}}$ & $318.9 \pm 18.9^{\mathrm{C}}$ \\
Total tannins (mg GAE / g) & $440.0 \pm 13.4^{\mathrm{A}}$ & $611.5 \pm 16.4^{\mathrm{B}}$ & $132.2 \pm 13.2^{\mathrm{C}}$ \\
Condensed tannins (mg CE / g) & $9.00 \pm 0.04^{\mathrm{A}}$ & $11.50 \pm 0.05^{\mathrm{B}}$ & $2.70 \pm 0.03^{\mathrm{C}}$ \\
\hline
\end{tabular}

GAE: Gallic Acid Equivalent; CE: Catechin Equivalent. In each line different letters mean significant differences $(p<0.05)$.

Alasalvar [17] used Sephadex LH-20 chromatography to separate crude extracts of hazelnut skin into low-molecular-weight polyphenols (Fraction One) and tannins (Fraction Two) with 95\% ethanol and $50 \%$ acetone as mobile phases, respectively. The total polyphenols content in crude extract, Fractions One and Two was $686 \pm 7,441 \pm 12$, and $697 \pm 11 \mathrm{mg} / \mathrm{g}$, respectively. This means the polyphenols contents in Fraction Two were approximately 1.58-fold higher than those in Fraction One, and 1.02-fold higher than those in crude extract. Therefore, the isolation of polyphenols and tannins using Sephadex LH-20 chromatography seemed less effective than the macroporous resin chromatography used in the present study. Ogawa [7] used Diaion HP-20, Chromatorex ODS 1024T, and Sephadex LH-20 chromatography to fractionate the seed shells of Aesculus turbinate, and highly purified polyphenols fractions were obtained. However, three kinds of chromatography were used including macroporous resin, normal-phase and reverse-phase chromatography, which was very complicated and too much toxic methanol was needed. Therefore, this strategy seems much more suitable for analysis than for industrial application. The one-step HPD-100 macroporous resin chromatography procedure established here provided an effective, low cost and simple method for chestnut industries to extract and purify polyphenols or tannins from Chinese chestnut spiny burs, which would facilitate the utilization of the resources of chestnut burs discarded as a waste conventionally.

\subsection{Scavenging of DPPH and ABTS Radicals}

The DPPH and ABTS radical scavenging assays are usually employed to evaluate the ability of antioxidants to scavenge free radicals. As the reaction between antioxidant molecules and radicals progresses, the absorbance of the reaction system decreases. Hence, the change of absorbance is used as a measure for the scavenging of DPPH and $\mathrm{ABTS}^{+}$radicals, and the more rapidly the absorbance changes, the more potential antioxidant activity the samples possess [17]. In the present study, all four different solvent extracts of chestnut burs exhibited considerable DPPH radical scavenging activities, and no significant difference was observed between $30 \%, 50 \%$ and $75 \%$ ethanol extracts (Figures $1 \mathrm{~A}$ and $2 \mathrm{~A}$ ). However, the DPPH and $\mathrm{ABTS}^{+}$radical scavenging activity of water extract was lower than the others. Similar results were observed for $\mathrm{EC}_{50}$ values (Table 3), which are defined as micrograms of extracts or fractions per assay required for $50 \%$ scavenging of DPPH and $\mathrm{ABTS}^{+}$radicals. For four kinds of extracts, $75 \%$ ethanol extract showed the lowest $\mathrm{EC}_{50}$, indicating it possesses the greatest antiradical activity, followed by $50 \%$ ethanol, $30 \%$ ethanol and water extracts. With respect to the fractions, Fraction II showed higher radicals scavenging activities than crude extract and Fraction I (Figures 1B and 2B), which indicated that Fraction II was effectively enriched in constituents responsible for radicals scavenging activity after macroporous resin chromatography. 
Figure 1. DPPH radical scavenging activity (RSA) of C. mollissima burs extracts (A) and fractions (B).
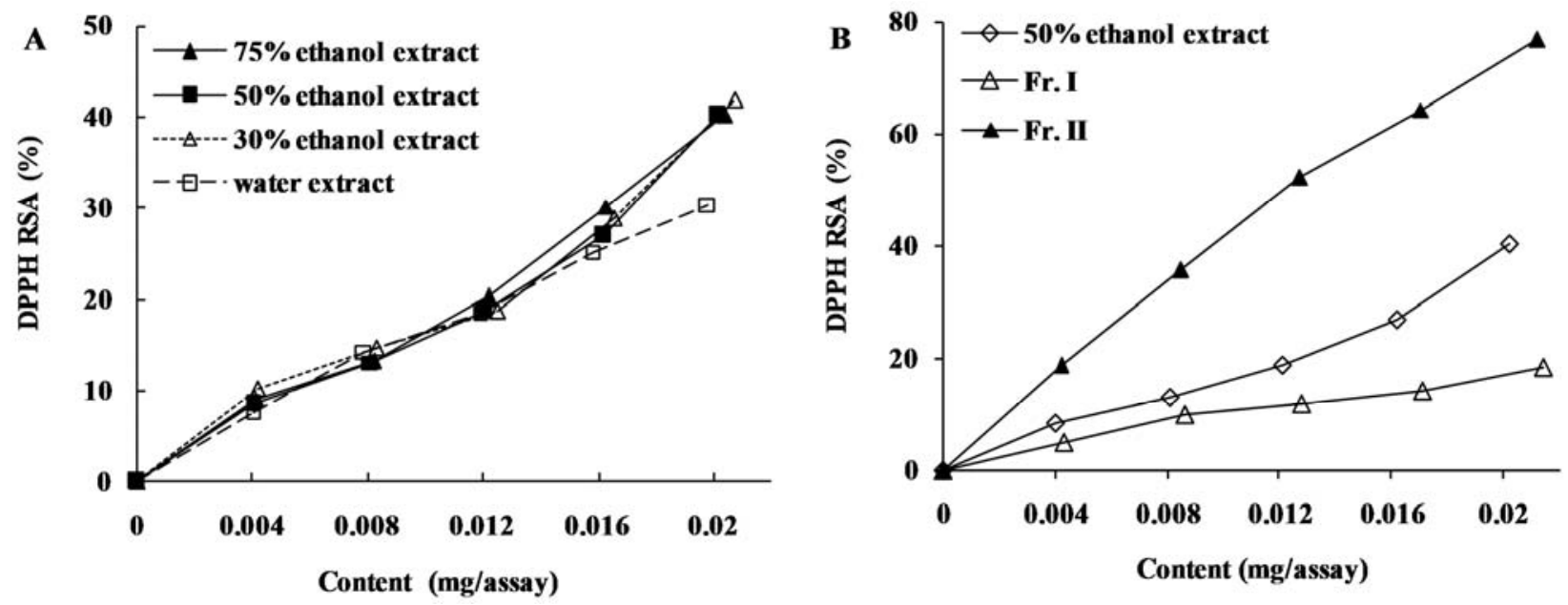

Figure 2. ABTS radical scavenging activity (RSA) of C. mollissima burs extracts (A) and fractions (B).
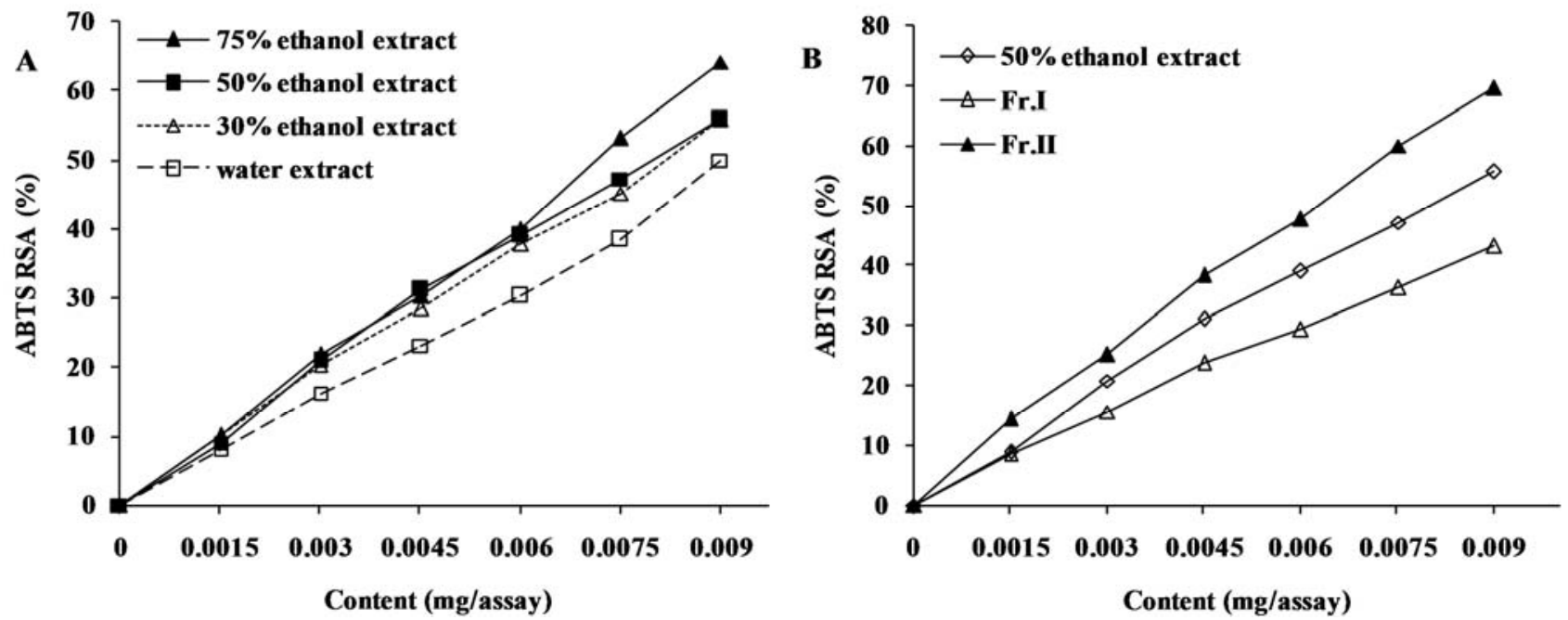

Table 3. Antiradical activities in chestnut burs extracts and fractions.

\begin{tabular}{ccccccc}
\cline { 2 - 7 } & $\begin{array}{c}\text { Water } \\
\text { extraction }\end{array}$ & $\begin{array}{c}\mathbf{3 0 \%} \text { Ethanol } \\
\text { extraction }\end{array}$ & $\begin{array}{c}\mathbf{5 0 \%} \text { Ethanol } \\
\text { extraction }\end{array}$ & $\begin{array}{c}\mathbf{7 5 \%} \text { Ethanol } \\
\text { extraction }\end{array}$ & $\begin{array}{c}\text { Fraction } \\
\text { II }\end{array}$ & $\begin{array}{c}\text { Fraction } \\
\text { I }\end{array}$ \\
\hline DPPH & 50.9 & 36.0 & 35.9 & 33.8 & 11.4 & 188 \\
ABTS & 10.6 & 8.48 & 8.03 & 7.03 & 5.80 & 12.5 \\
\hline
\end{tabular}

\subsection{Reducing Power}

The reducing power of the crude extracts and their fractions of chestnuts burs is depicted in Figure 3. The absorbance of the reaction system was highly correlated with the concentration of extracts $\left(\mathrm{R}^{2}>0.97\right)$, and the higher slope of the line indicated higher reducing power of the samples. In comparing the $30 \%, 50 \%$ and $75 \%$ ethanol extracts, the slope of water extracts was the smallest, and the slopes of $30 \%, 50 \%$ and $75 \%$ ethanol extracts were almost the same $(p>0.05)$. With respect to 
Fraction I and Fraction II, the slope of Fraction II was much greater than both the crude extract and Fraction I. The result of reducing power assay is highly consistent with that of DPPH and ABTS radical scavenging assay.

Figure 3. Reducing power of C. mollissima burs extracts (A) and fractions (B).
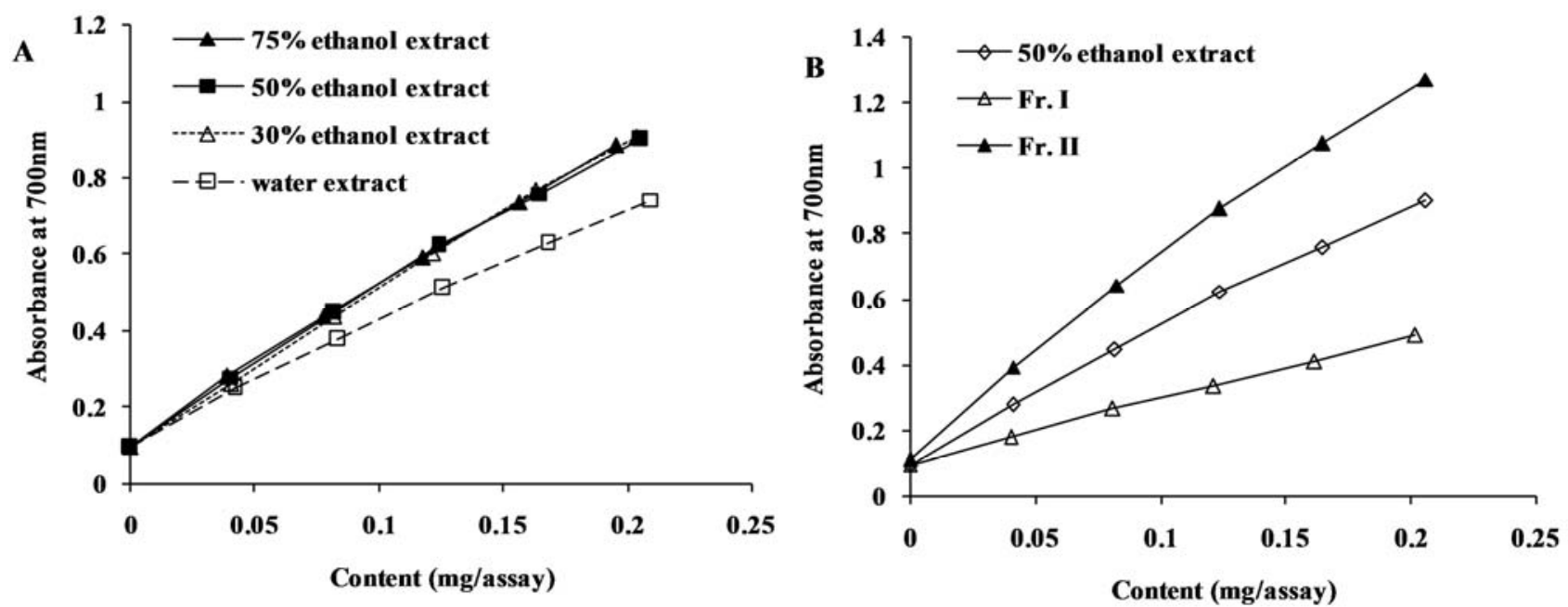

\subsection{Cellular Antioxidant Activity}

The cellular antioxidant activity (CAA) assay was developed by Liu to measure the antioxidant activity of antioxidants in cell culture in response to a need for a more biologically representative method than the chemical antioxidant activity assays as mentioned above including DPPH, ABTS radical scavenging assay and reducing power assay [17]. The CAAs of extracts and fractions of chestnut burs were measured with two protocols (PBS wash and no PBS wash), as described in literature [18]. The $\mathrm{EC}_{50}$, CAA values and median cytotoxicity doses are listed in Table 4.

Table 4. Cellular antioxidant activity of chestnut burs extracts and fractions.

\begin{tabular}{cccccc}
\hline & \multicolumn{2}{c}{ no PBS wash } & \multicolumn{2}{c}{ PBS wash } & cytotoxicity \\
\cline { 2 - 6 } & $\mathbf{E C}_{\mathbf{5 0}}$ & $\begin{array}{c}\text { CAA } \\
(\boldsymbol{\mu m o l} \mathbf{~ o f}\end{array}$ & $\begin{array}{c}\mathbf{E C}_{\mathbf{5 0}} \\
(\mathbf{m g} / \mathbf{m L})\end{array}$ & $\begin{array}{c}\text { CAA } \\
(\boldsymbol{\mu} \mathbf{m o l} \mathbf{~ o f} \\
\mathbf{Q E E} / \mathbf{1 0 0 g})\end{array}$ & $\begin{array}{c}\mathbf{C C}_{\mathbf{5 0}} \\
(\mathbf{m g} / \mathbf{M I})\end{array}$ \\
\hline water extraction & $38.7 \pm 3.5^{\mathrm{A}}$ & $11.6 \pm 0.4^{\mathrm{A}}$ & $389 \pm 34^{\mathrm{A}}$ & $1.86 \pm 0.29^{\mathrm{A}}$ & 249.3 \\
$30 \%$ ethanol extraction & $36.5 \pm 2.9^{\mathrm{A}}$ & $11.9 \pm 0.6^{\mathrm{A}}$ & $334 \pm 22^{\mathrm{B}}$ & $1.98 \pm 0.26^{\mathrm{A}}$ & 213.5 \\
$50 \%$ ethanol extraction & $33.5 \pm 3.1^{\mathrm{A}}$ & $12.6 \pm 0.5^{\mathrm{A}}$ & $276 \pm 19^{\mathrm{C}}$ & $2.86 \pm 0.23^{\mathrm{B}}$ & 224.9 \\
$75 \%$ ethanol extraction & $34.6 \pm 3.3^{\mathrm{A}}$ & $12.3 \pm 0.3^{\mathrm{A}}$ & $266 \pm 12^{\mathrm{C}}$ & $2.71 \pm 0.15^{\mathrm{B}}$ & 306.5 \\
Fraction I & $71.6 \pm 4.1^{\mathrm{B}}$ & $10.2 \pm 0.4^{\mathrm{B}}$ & $556 \pm 48^{\mathrm{D}}$ & $1.11 \pm 0.11^{\mathrm{C}}$ & 300.4 \\
Fraction II & $14.2 \pm 2.2^{\mathrm{C}}$ & $54.2 \pm 2.6^{\mathrm{C}}$ & $109 \pm 12^{\mathrm{E}}$ & $5.66 \pm 0.20^{\mathrm{D}}$ & 278.5 \\
\hline
\end{tabular}

QE: quercetin equivalent. In each column different letters mean significant differences $(p<0.05)$.

In the no PBS wash protocol CAA assay, the 50\% ethanol extraction had the highest CAA values and lowest $\mathrm{EC}_{50}$ values in the four kinds of extractions, followed by $75 \%$ ethanol, $30 \%$ ethanol and water extractions. However, the CAA values for four kinds of extracts were not significantly different from each other. In the PBS wash protocol, the same trend for CAA values was observed. With respect 
to $\mathrm{EC}_{50}$ in $\mathrm{CAA}$ assay with $\mathrm{PBS}$ wash protocol, the $75 \%$ ethanol extract showed the lowest values, followed by $50 \%, 30 \%$ ethanol and water extract, but no significant difference was observed between $50 \%$ and $75 \%$ ethanol extracts.

As illustrated in Table 4, in both PBS wash and no PBS wash protocol CAA assay, Fraction II exhibited significant higher CAA values and lower $\mathrm{EC}_{50}$ values than $50 \%$ ethanol extracts and Fraction $\mathrm{I}$. These results indicated that macroporous resin chromatography significantly enhanced not only the chemical antioxidant activity, but also the cellular antioxidant activity of chestnut burs in Fraction II.

\subsection{Correlation Analyses}

Using regression analyses, the relationships between total phenolic content, total tannins content and $\mathrm{EC}_{50}$ of DPPH and ABTS radicals scavenging values, as well as CAA values were determined. Total polyphenols were significantly negatively correlated to $\mathrm{EC}_{50}$ values of $\mathrm{DPPH}$ radicals scavenging $\left(\mathrm{R}^{2}=0.550, p<0.05\right)$ and ABTS radicals scavenging $\left(\mathrm{R}^{2}=0.764, p<0.05\right)$. Total tannins content was more significantly negatively correlated to $\mathrm{EC}_{50}$ values of DPPH radicals scavenging $\left(\mathrm{R}^{2}=0.598, p<0.05\right)$, and ABTS radicals scavenging $\left(\mathrm{R}^{2}=0.817, p<0.05\right)$ than those of poly-phenols. Total polyphenols were significantly positively correlated to CAA values $\left(\mathrm{R}^{2}=0.403, p<0.05\right.$ for the no PBS wash protocol; $\mathrm{R}^{2}=0.515, p<0.05$ for PBS wash protocol). Total tannins content was also significantly positively correlated to CAA values $\left(\mathrm{R}^{2}=0.452, p<0.05\right.$ for no PBS wash protocol; $\mathrm{R}^{2}=0.512, p<0.05$ for PBS wash protocol). These correlation analyses revealed that the antioxidant activity of the chestnut burs extracts and fractions was highly correlated with the content of phenolic and tannins in the chestnut burs.

\section{Experimental}

\subsection{Plant Samples and Reagents}

The Chinese chestnut burs were harvested at a chestnut plantation in Qianxi, Hebei Province of China at the beginning of the harvest season of 2008, authenticated by Dr Yujun Liu, Beijing Forestry University. The burs were air-dried till equilibrium humidity, ground and transferred to darkness for further use. All chemicals were purchased from Sigma-Aldrich, Inc. unless otherwise specified. HepG2 human liver cancer cells were kindly offered by Professor Du Lijun, Tsinghua University, China.

\subsection{Extraction of Chestnut Burs Polyphenols and Tannins}

The chestnut burs powder was extracted using four different solvent systems [water, 30:70 (v/v) ethanol/water, 50:50 (v/v) ethanol/water and 75:25 (v/v) ethanol/water] at a solid to solvent ratio of 1:10 (w/v), and subsequently placed in a shaking constant-temperature water bath at $80{ }^{\circ} \mathrm{C}$ for $1 \mathrm{~h}$. The resulting slurries were centrifuged at $5,000 \mathrm{~g}$ for $10 \mathrm{~min}$ to obtain the supernatant. The residues were subjected to repeated extraction twice under the same condition and the extracts supernatants were collected, combined and then evaporated under vacuum at $50{ }^{\circ} \mathrm{C}$. After concentrated, the extract was lyophilized at $-50{ }^{\circ} \mathrm{C}$ and $0.1 \mathrm{MPa}$, and finally restored at $-20{ }^{\circ} \mathrm{C}$ in vacuum-sealed colored pouches for further analysis. The extraction yields are expressed by reference to dry matter. 


\subsection{Fractionation of Chestnut Bur Extracts}

The chestnut burs were extracted with $50 \%$ ethanol according to the methods described above, and the supernatants were combined after centrifugation and evaporated till $20 \%$ volume left. Then, the extracts were replenished with distilled water to the initial volume and centrifuged at 5,000 g for $10 \mathrm{~min}$ to obtain the supernatant. Subsequently, the supernatant was subjected to a column chromatography (600 $\mathrm{mm} \times 60 \mathrm{~mm}$ i.d.) packed with HPD 100 macroporous resin (Cangzhou Bonchem Co., Ltd, Cangzhou,China) and equilibrated with distilled water. Fraction I was eluted with $2,000 \mathrm{~mL}$ distilled water, and Fraction II was obtained after elution with $2,000 \mathrm{~mL}$ of $50 \%$ ethanol as mobile phase. Both Fraction I and Fraction II were evaporated, lyophilized and finally stored at $-20{ }^{\circ} \mathrm{C}$ in darkness for further analysis.

\subsection{Determination of the Content of Total Polyphenols, Total Tannins and Condensed Tannins}

The content of total polyphenols was estimated using the Folin-Ciocalteu's phenol reagent as described by Amarowicz et al. [19]. The content of total tannins was determinate based on phosphomolybdium tungstic acid-casein reaction, described in Chinese Pharmacopoeia 2005 [20]. Briefly, sample solution $(25 \mathrm{~mL})$ was quickly mixed with casein $(0.6 \mathrm{~g})$ and incubated at $30^{\circ} \mathrm{C}$ for $1 \mathrm{~h}$. After filtration, the supernatant was collected. Then, the polyphenols content in original solution and supernatants were assessed using Folin-Ciocalteu's phenol reagent, and the total tannins were defined as polyphenols which could be absorbed by casein. The total polyphenols and total tannins results were expressed as g GAE / $100 \mathrm{~g}$ extract (on a dried weight basis). The content of condensed tannins was determined according to a vanillin/ $\mathrm{HCl}$ method [21], and the results were expressed as $\mathrm{g} \mathrm{CE}$ (Catechin Equivalent) / $100 \mathrm{~g}$ extract (on a dried weight basis).

\subsection{Determination of DPPH and ABTS Radical Scavenging Activity}

The method described by Alasalvar [17] was used to assess DPPH and ABTS radicals scavenging activities of the extracts and fractions. For DPPH radicals assay, aqueous solutions $(0.1 \mathrm{~mL})$ of chestnut bur extracts or fractions with concentration of $40-200 \mu \mathrm{g} / \mathrm{mL}$ were mixed with a freshly prepared DPPH solution $(0.25 \mathrm{~mL}, 1 \mathrm{mM}$ in methanol) and ethanol $(2 \mathrm{~mL})$. The mixture was shaken vigorously and left to stand for $20 \mathrm{~min}$ in the dark at room temperature and the absorbance was read at $517 \mathrm{~nm}$. For ABTS radicals assay, an ABTS solution $(7 \mathrm{mM})$ was mixed with potassium persulfate $(140 \mathrm{mM})$ with a ratio of $62.5: 1$ for $16 \mathrm{~h}$ in the darkness at room temperature to produce ABTS radical cation $\left(\mathrm{ABTS}^{+}\right)$stock solutions. The $\mathrm{ABTS}^{+}$stock solution was diluted with ethanol to an absorbance of 0.70 at $734 \mathrm{~nm}$ as a working solution. An aliquot $(0.15 \mathrm{~mL})$ of aqueous solution containing 10 to $60 \mu \mathrm{g} / \mathrm{mL}$ of chestnut bur extract or fraction was mixed thoroughly with the $\mathrm{ABTS}^{+}$solution $(2.85 \mathrm{~mL})$ and after 6-10 $\mathrm{min}$ in the darkness at room temperature; the absorbance was read at $734 \mathrm{~nm}$. The radicalscavenging activity (RSA) for DPPH and ABTS radicals was calculated as $\% R S A=100\left(A_{0}-A_{s}\right) / A_{0}$, where $A_{s}$ is the absorbance of the extract solution and $A_{0}$ is the absorbance of a control solution prepared without extracts. 


\subsection{Determination of Reducing Power}

The method described by Oyaizu [22] was used to assess the reducing power of the extract and its fractions. Briefly, an aliquot $(1 \mathrm{~mL})$ of aqueous solution containing 0.04 to $0.2 \mathrm{mg}$ extract or its fractions was mixed with $0.2 \mathrm{M}$ phosphate buffer $(2.5 \mathrm{~mL}, \mathrm{pH} 6.6)$ and $1 \%(\mathrm{w} / \mathrm{v})$ solution of potassium ferricyanide $\left[\mathrm{K}_{3} \mathrm{Fe}(\mathrm{CN})_{6}\right](2.5 \mathrm{~mL})$. After incubation in water bath at $50{ }^{\circ} \mathrm{C}$ for $20 \mathrm{~min}, 10 \%(\mathrm{w} / \mathrm{v})$ trichloroacetic acid solution $(2.5 \mathrm{~mL})$ was added, and then the mixture was incubated at room temperature for $10 \mathrm{~min}$. Subsequently, the mixture $(2.5 \mathrm{~mL})$ was combined with distilled water $(2.5 \mathrm{~mL})$ and $0.1 \%(\mathrm{w} / \mathrm{v})$ solution of ferric chloride $\left(\mathrm{FeCl}_{3}\right)(0.5 \mathrm{~mL})$. Finally, absorbance of the reaction mixture was recorded at $700 \mathrm{~nm}$. Results were expressed as the concentration of extract or fractions per assay versus absorbance at $700 \mathrm{~nm}$.

\subsection{Determination of Cellular Antioxidant Activity}

The cellular antioxidant activity (CAA) was determined using the protocol established by Liu et al. [18,23]. Briefly, complete medium (Williams' Medium E supplemented with 5\% fetal bovine serum, $10 \mathrm{mM}$ Hepes, $2 \mathrm{mM}$ L-glutamine, $5.0 \mu \mathrm{g} / \mathrm{mL}$ insulin, $0.05 \mu \mathrm{g} / \mathrm{mL}$ hydrocortisone, 50 units $/ \mathrm{mL}$ penicillin, $50 \mu \mathrm{g} / \mathrm{mL}$ streptomycin, and $100 \mu \mathrm{g} / \mathrm{mL}$ gentamycin) was used as growth medium for human HepG2 liver cancer cells. Then, cells were seeded on a 96-well microplate at a density of $6 \times 10^{4} /$ well in $100 \mu \mathrm{L}$ of growth medium/well. Twenty-four hours later, the growth medium was removed, and all wells were washed with phosphate buffered saline (PBS, $100 \mu \mathrm{L}$ ). After washing, wells were treated with $100 \mu \mathrm{L}$ solvent control, quercetin control, or tested samples plus $25 \mu \mathrm{M}$ DCFH-DA (2',7'-dichlorofluorescin diacetate) for $60 \mathrm{~min}$. Then cells were treated with Hanks' Balanced Salt Solution $(100 \mu \mathrm{L})$ with $10 \mathrm{mM}$ Hepes and $600 \mu \mathrm{M}$ 2.2'-azobis(2-amidinopropane) (ABAP). Finally, emission at $538 \mathrm{~nm}$ was measured after excitation at $485 \mathrm{~nm}$ with a Fluoroskan Ascent FL plant reader at $37{ }^{\circ} \mathrm{C}$ every $5 \mathrm{~min}$ for $60 \mathrm{~min}$. The $\mathrm{EC}_{50}$ (median effective dose) was determinated for extracts and fractions as described in literature, and $\mathrm{EC}_{50}$ values were converted to CAA values expressed as micromoles of quercetin equivalents per $100 \mathrm{~g}$ extracts [24]. The cytotoxicity of each extraction or fraction was determinate as described in literatures [18,23].

\subsection{Statistical Analysis}

Values are presented as mean \pm standard deviation $(\mathrm{SD})(\mathrm{n}=3)$ for each extract and fractions. The statistical significance ( $t$-test: two-sample equal variance, using two-tailed distribution) was determined using Microsoft Excel statistical software (Microsoft Office Excel 2007, Microsoft Corp. Redmond, WA, USA). Differences at $p<0.05$ were considered to be significant.

\section{Conclusions}

The present study investigated the extraction and isolation process of chestnut burs tannins, as well as the tannins' chemical and biological activity as natural antioxidants and antiradicals. An effective, low cost and simple procedure for tannin extraction and isolation using 50\% ethanol as extraction solvent and one-step HPD-100 macroporous resin chromatography with 50\% ethanol as elution solvent was established. The phenol and tannin enriched extracts obtained using this method exhibited 
considerable DPPH and ABTS radical scavenging activity, reducing power, and cellular antioxidant activity. The fraction responsible for antioxidant activities in Chinese chestnut burs was primarily characterized as hydrolysable tannins. To the best of our knowledge, this is the first report of enrichment of tannins from burs of $C$. mollissim using macroporous resin chromatography, and to assess their cellular antioxidant activity. This study extends our knowledge about the potential bioactivities and applications in cosmetic, pharmaceutical and food processing industries of tannins abundant in the chestnut burs.

\section{Acknowledgments}

The study was financially supported by the Fundamental Research Funds for the Central Universities, China (BLYX200922) and the Ph.D. Programs Foundation of Ministry of Education of China (20100014120017). Also, we are grateful to Jian-zhong Wang and Feng-Jun Wang, Beijing Forestry University, for providing chestnut burs sample.

\section{References and Notes}

1. Zhang, D.S.; Gao, H.Y.; Wang, L.B.; Li, D.; Kuroyanagi, M.; Wu, L.J. Flavonol glycosides from Castanea mollissima Blume. Asian J. Trad. Med. 2007, 2, 229-234.

2. Kidd, P.M. Bioavailability and activity of phytosome complexes from botanical polyphenols: The silymarin, curcumin, green tea, and grape seed extracts. Altern. Med. Rev. 2009, 14, 226-246.

3. Adams, L.S.; Zhang, Y.; Seeram, N.P.; Heber, D.; Chen, S. Pomegranate ellagitannin-derived compounds exhibit antiproliferative and antiaromatase activity in breast cancer cells in vitro. Cancer. Prev. Res. 2010, 3, 108-113.

4. Huang, W.Y.; Cai, Y.Z.; Zhang, Y. Natural phenolic compounds from medicinal herbs and dietary plants: potential use for cancer prevention. Nutr. Cancer 2010, 62, 1-20.

5. Suzuki, J.; Isobe, M.; Morishita, R.; Nagai, R. Tea polyphenols regulate key mediators on inflammatory cardiovascular diseases. Mediat. Inflamm. 2009, Article ID 494928, 5 pages.

6. Magrone, T.; Candore, G.; Caruso, C.; Jirillo, E.; Covelli, V. Polyphenols from red wine modulate immune responsiveness: Biological and clinical significance. Curr. Pharm. Des. 2008, 14, 2733-2748.

7. Ogawa, S.; Kimura, H.; Niimi, A.; Katsube, T.; Jisaka, M.; Yokota, K. Fractionation and structural characterization of polyphenolic antioxidants from seed shells of Japanese horse chestnut (Aesculus turbinata BLUME). J. Agric. Food Chem. 2008, 56, 12046-12051.

8. Vázquez, G.; Fontenla, E.; Santos, J.; Freire, M.S.; González-Alvarez, J.; Antorrena, G. Antioxidant activity and phenolic content of chestnut (Castanea sativa) shell and eucalyptus (Eucalyptus globulus) bark extracts. Ind. Crops Prod. 2008, 28, 279-285.

9. You, Y.; Duan, X.; Wei, X.; Su, X.; Zhao, M.; Sun, J.; Ruenroengklin, N.; Jiang, Y. Identification of major phenolic compounds of Chinese water chestnut and their antioxidant activity. Molecules 2007, 12, 842-852.

10. Vázquez, G.; González-Álvarez, J.; Freire, M.S.; Fernández-Agulló, A.; Santos, J.; Antorrena, G. Chestnut burs as a source of natural antioxidants. In Proceedings of The Ninth International Conference on Chemical and Process Engineering, Rome, Italy, 10-13 May 2009. 
11. Vázquez, G.; Fernández-Agulló, A.; Gómez-Castro, C.; Freire, M.S.; Antorrena, G.; González-Álvarez, J. Response surface optimization of antioxidants extraction from chestnut (Castanea sativa) bur. Ind. Crops Prod. 2012, 35, 126-134.

12. Mujić, A.; Grdović, N.; Mujić, I.; Mihailović, M.; Živković, J.; Poznanović, G.; Vidaković, M. Antioxidative effects of phenolic extracts from chestnut leaves, catkins and spiny burs in streptozotocin-treated rat pancreatic $\beta$-cells. Food Chem. 2011, 125, 841-849.

13. Zhao, Y.; Chen, B.; Yao, S. Separation of 20(S)-protopanaxdiol type ginsenosides and 20(S)protopanaxtriol type ginsenosides with the help of macroporous resin adsorption and microwave assisted desorption. Sep. Purif. Tech. 2007, 52, 533-538.

14. Silva, E.M.; Pompeu, D.R.; Larondelle, Y.; Rogez, H. Optimisation of the adsorption of polyphenols from Inga edulis leaves on macroporous resins using an experimental design methodology. Sep. Purif. Technol. 2007, 53, 274-280.

15. Sun, A.; Sun, Q.; Liu, R. Preparative isolation and purification of flavone compounds from Sophora japonica L. by high-speed counter-current chromatography combined with macroporous resin column separation. J. Sep. Sci. 2007, 30, 1013-1018.

16. Wünsch, P.; Del Vedovo, S.; Rosset, J.; Smiley, M. The tannin granules from ripe carob pod. Lebenson-Wiss. Technol. 1984, 17, 351-354.

17. Alasalvar, C.; Karamać, M.; Kosińska, A.; Rybarczyk, A.; Shahidi, F.; Amarowicz, R. Antioxidant activity of hazelnut skin phenols. J. Agric. Food Chem. 2009, 57, 4645-4650.

18. Wolfe, K.L.; Kang, X.; He, X.; Dong, M.; Zhang, Q.; Liu, R.H. Cellular antioxidant activity of common fruits. J. Agric. Food Chem. 2008, 56, 8418-8426.

19. Amarowicz, R.; Troszyńska, A.; Shahidi, F. Antioxidant activity of almond seed extract and its fractions. J. Food Lipids 2005, 12, 344-358.

20. Pharmacopoeia of the People's Republic of China, 2005 English Edition; Volume I, Appendix XB.

21. Takahata, Y.; Ohnishi-Kameyama, M.; Furuta, S.; Takahashi, M.; Suda, I. Highly polymerized procyanidins in brown soybean seed coat with a high radical-scavenging activity. J. Agric. Food Chem. 2001, 49, 5843-5847.

22. Oyaizu, M. Studies on products of the browning reaction. Antioxidative activities of products of browning reaction prepared from glucosamine. Jpn. J. Nutr. 1986, 44, 307-315.

23. Wolfe, K.L.; Kang, X.; He, X.; Dong, M.; Zhang, Q.; Liu, R.H. Cellular antioxidant activity of common fruits. J. Agric. Food Chem. 2008, 56, 8418-8426.

24. Song, W.; Derito, C.M.; Liu, M.K.; He, X.; Dong, M.; Liu, R.H. Cellular antioxidant activity of common vegetables J. Agric. Food Chem. 2010, 58, 6621-6629.

Sample Availability: Samples are available from the authors.

(C) 2011 by the authors; licensee MDPI, Basel, Switzerland. This article is an open access article distributed under the terms and conditions of the Creative Commons Attribution license (http://creativecommons.org/licenses/by/3.0/). 